\title{
Bird's Eye View on the Recent Advances in Drug Delivery Systems
}

\author{
Pramod Vishwanath Prasad ${ }^{1 *}$, Anand Kumar ${ }^{2}$, Pramod Chandra Pal ${ }^{3}$, Radhey Shyam Sharma ${ }^{4}$, \\ Donthamsetty Nageswara Rao ${ }^{3}$, Tulsidas G. Shrivastav ${ }^{5}$, Ren-Shan Ge ${ }^{1}$
}

${ }^{1}$ Center for Biomedical Research, Population Council, The Rockefeller University, New York, USA; ${ }^{2}$ Department of Reproductive Biology, All India Institute of Medical Sciences, New Delhi, India; ${ }^{3}$ Department of Biochemistry, All India Institute of Medical Sciences, New Delhi, India; ${ }^{4}$ Division of RHN, Indian Council of Medical Research, New Delhi, India; ${ }^{5}$ Department of Reproductive Biomedicine, National Institute of Health and Family Welfare, New Delhi, India.

E-mail: *drpramod.pusa@gmail.com, ${ }^{*}$ pkbiochem@yahoo.com

Received September $5^{\text {th }}, 2011$; revised October $14^{\text {th }}, 2011$; accepted November $20^{\text {th }}, 2011$.

\begin{abstract}
Successful treatment of most of the diseases is limited by a lack of safe and effective methods of drug delivery. Drug delivery methods have significant effects on the pharmacological efficacy of a drug. Every drug has an optimum concentration range within which maximum benefit is derived; and concentrations above or below the range can be toxic or provide no therapeutic benefits at all. Therefore, development of an efficient drug delivery system remains an important challenge in medicine, and this can be achieved only through multidisciplinary approaches to the mechanisms of delivery of drugs to targets in tissues. Thus, several drug delivery and drug targeting systems are currently being developed. Targeting is an ability to direct the drug(s) to the desired site. There are two major mechanisms, viz., active and passive for drug targeting. Controlled drug release and subsequent biodegradation are also indispensable for developing successful formulations. Colloidal drug vehicles such as micelles, vesicles, liquid crystal dispersions, and nanomaterials consisting of miniscule nanoparticles of 5 - $200 \mathrm{~nm}$ diameter have shown great promise as drug delivery systems. In this context, past decades have witnessed certain major advancements. This review article emphasizes on these advances in the field of drug delivery systems.
\end{abstract}

Keywords: Drug Delivery Vehicles, Nanoparticles, Colloidosomes, Dendrimers, Nanorobots

\section{Introduction}

Drug delivery system is a process of administering drug(s) to achieve its maximal therapeutic benefits in humans or animals. The main objective of developing new drug delivery systems is to promote the therapeutic effects of a drug by 1) increasing drug's bioavailability, 2) preventing its toxic, harmful side-effects by increasing the amount and persistence of a drug in the vicinity of target cell and reducing the drug exposure of non-target cells, and 3) minimizing drug degradation and loss [1-3]. Taking these challenges into account, various drug delivery and drug targeting systems are currently under development. There are, however, some drug delivery vehicles (carriers) viz., soluble polymers, micro- and nano-particles made up of insoluble or biodegradable natural and synthetic polymers, microcapsules, cells, micelles, liposomes etc. have been developed. The carriers can be made stimuli-reactive (for instance, $\mathrm{pH}$ - or temperature-sensitive), slowly degradable, and targeted (e.g., by coupling them with specific antibodies against certain characteristic components of the area of interest). Targeting is the ability to direct the drug-loaded system to the site of interest. There are two mechanisms viz., 1) passive and 2) active by which drug(s) are targeted (released) at the desired site(s). An example of passive targeting is the preferential accumulation of chemotherapeutic agents in solid tumors as a result of the enhanced vascular permeability of tumor tissues compared with healthy tissue; whereas active targeting involves the surface functionalization of drug carriers with ligands that are selectively recognized by receptors on the surface of the cells of interest. Since ligand-receptor interactions can be highly selective, this could allow a more precise targeting of the site of interest.

Drug delivery systems are based on physical and biochemical mechanisms. Physical mechanisms also re- 
ferred as controlled drug delivery systems. It includes: 1) desorption of adsorbed or surface-bound $\operatorname{drug}(\mathrm{s}), 2)$ diffusion through the carrier matrix, 3 ) diffusion (in the case of nanocapsules) through the carrier wall, 4) carrier matrix erosion, and 5) a combined erosion and diffusion process. Biochemical mechanisms involve monoclonal antibodies, gene therapy, vector systems, liposomes and polymer drug adducts. Sustained or continuous drug delivery systems involve polymer(s) that release drug(s) at a controlled rate due to diffusion out of the polymer(s) or by degradation of the polymer(s) over time. Drug delivery systems exhibiting pulsatile release of drug(s) closely mimics the way by which the body naturally produces certain hormones like cortisol, insulin etc. It is achieved by using drug-carrying polymers that respond to specific stimuli (such as, changes in $\mathrm{pH}$ or temperature, exposure to light etc). The mode of delivery can be the difference between a drug's success and failure, as the choice of a medicine is often influenced by the way the drug is administered.

In recent years, the emergence of nanotechnology has had a profound effect on many areas of healthcare and scientific research. Scientists have known the potential benefits of nanotechnology in providing remarkable improvements in drug delivery and drug targeting. Any improvement in delivery techniques which minimize toxicity and improve efficacy offers great potential benefits to patients. Attempts are being made to better target the drug and improve its effectiveness by 1) crossing particular physical barriers, like the blood-brain barrier, and 2) finding alternative and acceptable routes for the delivery of protein drugs other than the gastro-intestinal tract, where degradation can usually occur.

Pharmacological efficacy of any drug significantly depends on the methods of its delivery. Every drug has an optimum concentration range within which maximum benefit is obtained, and concentrations above or below the range can be harmful or provide no therapeutic benefits. Therefore, determination of optimum dose of any therapeutics and its efficient delivery to target tissues for successfully treating diseases needs a multidisciplinary approach. In this approach, several innovative and strategic ideas on controlling the pharmacokinetics, pharmacodynamics, non-specific toxicity, immunogenicity, biorecognition, and efficacy of drug(s) were generated and utilized. As well, the combined approaches from various disciplines viz., polymer science, pharmaceutics, bioconjugate chemistry, and molecular biology and highly integrated efforts of biologists, chemists, nanotechnologists, biochemical- and biophysical-engineers resulted into the development of several new drug delivery systems over the past decade. In the present review, an ex- tensive effort has been made to bring them together on a single platform, under following headings:

\section{Drug Delivery Vehicles}

Drug delivery vehicles are substances that serve as mechanisms to improve the delivery and the effectiveness of drug(s). Vehicles are also used in designs to increase the effectiveness of drug delivery to the target sites of pharmacological actions. The aims of developing drug delivery vehicles are to obtain systems with optimized drug loading and release properties, long shelf-life and low toxicity. Some of the recently developed drug delivery vehicles are briefed here.

1) Nanomaterials are hollow or solid structure, with diameter in the $1-1000 \mathrm{~nm}$ range, and can be filled or loaded with drugs and detection agents. Targeting moieties can also be attached to their surface to make them useful for targeted drug delivery. The nanomaterials include nanoparticles, lipid core micelles, polymeric micelles, biodegradable polymeric nanoparticles, liposomes, nanopores, nanospheres, nanocapsules, nanotubes, nanowires, nanoshells, nanocantilever, dendrimers, gold nanoparticles, protein nanoparticles, buckminsterfullerene (commonly called bucky balls) and quantum dots. It must be noted that all of the nanomaterials mentioned are commonly termed as 'nanoparticles', depending on the ease of user. However, nanoparticles include only solid entities.

2) Nanoparticles are either amorphous or crystalline solid. They are typically made up of a single material and measure about $5-200 \mathrm{~nm}$ in size. They have the property to absorb and (or) encapsulate drug(s), thus protecting it(them) against chemical and enzymatic degradation. They have emerged as potential drug delivery devices because of their applications in the controlled release of drugs [4]. They have been particularly employed in targeting particular tissues/organs as carriers of DNA in gene-therapy, and in their ability to deliver proteins, peptides, genes through oral route. Efforts for application of protein nanoparticles in drug delivery are being made [5]. Superparamagnetic iron oxide nanoparticles (SPIONs) are commonly produced by reduction of iron salts under alkaline condition. They have proved to be promising drug delivery vehicles for some biomedical applications such as targeted drug delivery and imaging, hyperthermia, magneto-transfections, gene therapy [6,7], stem cell tracking, molecular/cellular tracking, magnetic separation technologies (e.g. rapid DNA sequencing), and detection of liver and lymph node metastases. Most recently, SPIONs have been successfully used for early detection of inflammatory diseases, cancer, diabetes and atherosclerosis [8]. Currently, poly ethylene glycolpoly- 
hedral oligosilsesquioxane (PEG-POSS) amphiphilic nanoparticles are being investigated to encapsulate insulin as new drug delivery vehicles. Insulin release tests have indicated that insulin was well-protected inside PEG-POSS nanoparticles at gastric $\mathrm{pH}$ for $2 \mathrm{hrs}$, and was released at intestinal $\mathrm{pH}(\mathrm{pH} 6$ - 7) where the absorption and activation of the drug are necessary. Therefore, such nanostructured PEG-POSS nanoparticles seemed to be useful as a potential carrier for oral insulin drug delivery systems, in near future [9].

3) Lipid core micelles and polymeric micelles are nanosized colloidal particles. Both of them are made up of hydrophobic core and hydrophilic shell. They are successfully used for the solubilization of various poorly soluble drugs (particularly anticancer drugs) and demonstrate attractive properties as drug carriers for targeted drug delivery. Drug-loaded lipid-core micelles can spontaneously target body areas with compromised vasculature (viz., tumors, infarcts) via the enhanced permeability and retention (EPR) effect [1]. Biodegradable polymeric nanoparticles or microspheres are potential drug delivery carriers of peptide and protein drugs and vaccines [10].

4) On contrary to the lipid core micelles and polymeric micelles, liposomes are microscopic nanoparticles made up of phospholipid bilayers surrounding an aqueous core. The polar nature of liposomal core facilitates polar drug moieties to be encapsulated. However, amphiphilic and lipophilic drug moieties are also encapsulated by solubilizing them within phospholipid bilayer [11]. On the basis of size and lamellarity (number of bilayers present within a liposome), liposomes are classified into three types: small unilamellar vesicles (SUVs), large unilamellar vesicles (LUVs), and multilamellar vesicles (MLVs). Different types of liposomes are widely used for delivering certain vaccines, cancer treatment, and against infectious diseases. Liposomes treat cancer by encapsulating drugs, shielding healthy cells from drug's toxicity and prevent drug's concentration in vulnerable tissues like liver and kidneys. They also eliminate some side-effects (like nausea, hair loss etc.) of cancer treatment. Moreover, fusogenic liposomes have the potential to deliver drugs intracellularly and therefore, markedly enhance therapeutic activity [12-15].

5) Nanowires are nanoscale sensing wires. They can be coated with molecules such as antibodies to bind to proteins of choice and transmit their information through sensing electrodes to the computers [16]. They have applications in the early sensing of breast and ovarian malignancies. In the rapidly emerging field of nanoneurosciences, nanowired drug delivery using Titanium dioxide $\left(\mathrm{TiO}_{2}\right)$-based nanowires $(50-60 \mathrm{~nm})$ tagged with drugs have been found to enhance the neuroprotective efficacy of drugs in spinal cord injury following trauma
$[17,18]$. Therefore, the use of nanowires as drug carriers significantly enhances the delivery and therapeutic efficacy of drug(s). Silicon nanowire-coated beads (i.e., nanowire coatings) paired with epithelial physiology have shown significantly increased adhesion (almost 100-fold), in mucosal conditions. Without these bioadhesive delivery devices, complex compounds were undergoing typical degradation and clearing from mucosal tissues by the mucous layer [19]. Currently, a novel hybrid nano-materials such as iron nanowires are being developed for use in high thermal efficiency hyperthermia therapy [20].

6) Nanoshells are composite nanoparticles made up of a hollow silica core $\left(\mathrm{SiO}_{2}\right.$, semiconductor) surrounded by a gold shell $(\mathrm{Au})$. Silica-gold $\left(\mathrm{SiO}_{2}-\mathrm{Au}\right)$ nanoshells are unique because their peak extinctions are very easily tunable over a wide range of wavelengths, particularly in the near infrared region $(800-1200 \mathrm{~nm})$ of the spectrum. Light in this region is transmitted through tissue with relatively little attenuation due to absorption. In addition, irradiation of $\mathrm{SiO}_{2}-\mathrm{Au}$ nanoshells at their peak extinction coefficient results in the conversion of light to heat energy that produces a local rise in temperature. This makes nanoshell hot, consequently the heat kills the cancer cell. Thus, to develop a photothermal modulated drug delivery system, some groups of scientists have fabricated a nontoxic hydrogel polymer network encapsulating silicagold $\left(\mathrm{SiO}_{2}-\mathrm{Au}\right)$ nanoshells. The hydrogel coatings can be collapsed by exposure to light via excitation of the strong Plasmon resonance of the silica-gold nanoshells; this excitation provides a ready source of heat to the system. Photothermally modulated drug delivery profiles for methylene blue, insulin, and lysozyme have been achieved by irradiation of the drug-loaded nanoshell-composite hydrogels, which showed that drug release was dependent upon the molecular weight of the therapeutic molecule. Currently, these nanoparticles are further being developed as photothermally responsive drug-delivery vehicles that undergo structural changes slightly above physiological temperature [21-24]. Moreover, antibodies can be attached to the surfaces of nanoshells, thus enabling the shells to target certain cancer cells [4].

7) Nanotubes are hollow cylindrical tubes made up of carbon atoms, with cross-sectional dimensions in the nanometer range, and lengths can be extended over a thousand times their diameters. They can be filled with drug(s) and sealed and thus serve as potential drug delivery vehicles. Currently, Aluminium oxide nanotubes have been fabricated and found to have many favorable properties as drug carrier for controlled release of drug(s) [25]. It has potential for therapeutic surface coatings on medical implants, as well. Nanotubes have been reported as high-specificity sensors of antibody signatures of autoimmune disease [26] and of single-nucleotide poly- 
morphisms (SNPs) [16,27].

8) Nanobots are nanotubes intercalated with anticancer drug(s). They release drug(s) only near a tumor in response to $\mathrm{pH}$ stimulus, since tumors and inflammated places are typically more acidic $(\mathrm{pH} 6.5)$ than normal tissues (pH 7.4) [28].

9) Nanoneedles are hollow, tubular or conical needles in the nanometer size range. They are nontoxic, biodegradable and made up of eco-friendly materials silicon or boron-nitride. Nanoneedles have central bore of sufficient size to allow passage of molecules. They can be mounted in arrays and used to deliver any drug or vaccination antigen, straight through the cellular wall, either in the cytoplasm, or inside the very nucleus of the cell. The array is pressed against the skin. This may reduce the volume of antigen required to ensure adequate immunity, as well as creating multiple immune pathways. This system was found to deliver insulin and diclofenac effectively in the animal models [29]. Due to non-invasive penetration method, nanoneedles avoid pain sensation during drug or vaccine delivery (that will engender greater patient compliance). This simultaneously minimizes vaccine or drug degradation and enhances bioavailability. Nanoneedles serve as a powerful new tool for a) quantitatively examining biological processes occurring within a cell's nucleus or cytoplasm; b) studying biophysical properties at the molecular level inside living cells. Combined with molecular targeting strategies using quantum dots and magnetic nanoparticles as molecular probes, the nanoneedle drug delivery method can potentially enable the simultaneous observation and manipulation of individual molecules [30,31]. In future, nanoneedles can also be used as electrochemical probes and as optical biosensors to study cellular environments.

10) Nanorods are solid nanoneedles. They are also made up of silicon or boron-nitride. They are useful in light emitting diodes (LED), and laser diodes [29]. They have the potential to be used as drug delivery vehicles.

11) Nanocantilevers are made up of flexible beams of carbon atoms. Their beams are anchored at only one end, resembling a row of diving boards. The beams of nanocantilever deflect when the biomolecules of interest bind. The deflections are either observed directly by laser light or generate detectable shifts in the physical properties of the beam, such as their resonant-vibration frequency. Therefore, these biomolecular sensors have the ability to "multiplex" massively - that is, to detect a large number of different molecular species at the same time. Thus, the nanocantilevers (like microcantilevers) are being developed for serum and tissue proteomics-based cancer diagnostics, prognostics and therapeutic-efficacy monitoring $[16,32,33]$.

12) Nanopores are tiny holes engineered into particles.
The holes are engineered so tiny that DNA molecules can pass through them one strand at a time, allowing for highly precise and efficient DNA sequencing. Drug manufacturers can engineer nanopores into the surface of a drug capsule which are only slightly larger than the medicine's molecular size, thus controlling the rate of a drug's diffusion in the body. Currently, nanoporous systems engineered to mimic natural filtration systems are actively being developed for use in smart implantable drug delivery systems, bioartificial organs, and other novel nano-enabled medical devices. Synthetic nanoporous materials have numerous potential biological and medical applications that involve sorting, sensing, isolating, and releasing biological molecules [34]. Scientists have used mesoporous thin silica films with nanopores as anticancer drug carriers and gained mechanistic insights into the feasibility of using such films for drug delivery at the target-site [35]. Thus, nanoporous systems have applications in the cancer research and treatment.

13) Bucky balls (Fullerene) are common name given to a recently discovered molecule called buckminsterfullerene [36]. Bucky ball is made up of 60 carbon atoms arranged in a soccer ball-like structure. Unlike other molecules that have applications as cancer drug delivery vehicles, fullerenes do not break down in the body and are excreted intact. This trait helps for some cancer treatment compounds that are dangerous to healthy cells. For example, fullerene drug delivery particles that contain radioactive atoms would allow for the complete removal of radiation from the body following treatment [37]. Bucky balls and other fullerenes because of their chemistry and their unusual hollow, cage-like shape extremely stable and can withstand very high temperatures and pressures. Bucky ball is the only molecule composed of a single element to form a hollow spheroid which gives the potential for filling it, and using it as novel drug delivery systems. Bucky balls have some demerits like: it hurts cells, have high potential to accumulate in living tissues, and difficulty in targeting drug delivery site. But it may find global use in future products and applications like:

a) drug delivery vehicles for antitumor drugs or cancer therapy,

b) to fight allergy,

c) as inhibitor of HIV, and

d) as powerful anti-oxidant, and

e) in ultra-hard coating in the military services.

14) Gold Nanoparticles are made up of solid cores of Gold $(\mathrm{Au})$. Scientists are using gold nanoparticles to develop ultrasensitive detection systems for DNA diagnostics, biosensors and protein markers associated with many forms of cancer, including breast and prostate cancer [38]. Colloidal gold nanoparticles represent a com- 
pletely novel technology in the field of particle-based tumor-targeted drug delivery. Recently, it has been successfully used for targeted delivery of tumor necrosis factor to treat a solid tumor growing in mice [39].

15) Quantum Dots are miniscule semiconductor nanoparticles with an inert, non-toxic polymer coating. The core material can be chosen depending on the emission wavelength range being targeted. Targeting molecules can be attached to the coating. They serve as sign posts of certain types of cells or molecules in the body because they emit different wavelengths of radiations depending upon the type of Cadmium used in their cores. Cadmium sulfide (CdS) for ultra violet to blue, Cadmium selinide (CdSe) for most of the visible spectrum and Cadmium telluride (CdTe) for far-infra red and near infra red. Quantum dot bioconjugates with targeting antibodies have been used to recognize molecular signatures including ERBB2 [16,40,41]. Quantum dots are very stable under complex in vivo conditions that make them suitable for advanced molecular and cellular imaging, drug delivery and for highly sensitive bioassays and diagnostics [42-45]. Advances in the quantum dot technology can provide a great deal of information about the molecular events in tumor cells and early diagnosis of cancer.

16) Carbon nanotubes (CNT) are hollow nanostructures made up of carbon. They can be covalently or non-covalently modified to circulate well within the body. Such modifications can increase or decrease circulation time with in the body. CNT can be functionalised with bioactive peptides, proteins, nucleic acids and drugs, and used to deliver their cargos to cells and organs. Because functionalised CNT display non-significant toxicity and are non-immunogenic, therefore, they hold great potential as drug delivery vehicles and have emerged as an efficient nanovector for transporting and translocating therapeutic molecules [46-48]. Scientists have used CNT to deliver active agents, drugs in the animals. Carbon nanotubes have been developed to seek and destroy tumor cells [49].

17) Nanorobots are capable of treatment, prophylaxis, and diagnosis of certain ailments. They represent the next generation of drug delivery devices [50-52]. Coming era is most likely to see its usefulness in treating and diagnosing certain diseases.

18) Dendrimers are synthetic nanovectors (nanoparticles) measuring about $5-10 \mathrm{~nm}$ in diameter. They are made up of polymers with a central core. Dendrimers render drug molecules a greater water-solubility, bioavailability, and biocompatibility. They are hyperbranched (like a tree) and monodisperse three-dimensional molecules with defined molecular weights and have large numbers of functional groups to which drugs (and other molecules) can be attached. They have well-established host-guest entrapment properties. As the dendrimers have many different functional groups (sites), therefore drugs, and other molecules like polyethylene glycol can be attached. Polyethylene glycol attached to dendrimer prevents the body's immune system to detect it as foreign, thereby slows the process of breakdown of "drug loaded dendrimers". This fascinating nanovector holds significant promise for cancer diagnosis and treatment. Recently, scientists have fashioned dendrimers into sophisticated anticancer machines carrying five molecules as five different tools: a) a molecule designed to bind to cancer cells, b) second molecule that fluoresce upon locating genetic mutations, c) a third to assist in imaging tumor shape using X-rays, d) a fourth carrying drugs released on demand, and e) a fifth that would send a signal when cancerous cells are eventually dead. Therefore, dendrimers have significant promise as delivery vehicles for drugs, particularly, the non-steroidal anti-inflammatory, anti-microbial/anti-viral and potent anti-cancer drugs. A new generation of dendrimer-based delivery systems will enable the efficient transport of drugs across cellular barriers [53,54].

19) Hydrogels are enviro-intelligent and stimuli-sensitive three-dimensional gel systems [55]. They are made up of hydrophilic and polymeric networks capable of imbibing large amounts of water or biological fluids. The networks are composed of homopolymers or copolymers, and are insoluble due to the presence of chemical crosslinks (tie-points, junctions), or physical crosslinks, such as crystallites or entanglements. Hydrogels exhibit a thermodynamic compatibility with water, which allows them to swell in aqueous media. They are used to regulate drug release in reservoir-based, controlled release systems or as carriers in swellable and swelling-controlled release devices. As they are stimuli-sensitive so modulate release of drugs in response to electric field, temperature, $\mathrm{pH}$, ionic strength, and specific analyte concentration differences. In these controlled drug delivery systems, release can be made to occur within specific areas of the body (e.g., within a certain $\mathrm{pH}$ of the digestive tract) or also via specific sites (adhesive or cell-receptor specific gels via tethered chains from the hydrogel surface). Hydrogels as drug delivery systems can be very promising materials if combined with the technique of molecular imprinting (nanoscale templating). Chitosan is a cationic polymer (polysaccharide). It is gaining importance in the development of mucoadhesive hydrogelbased drug delivery system because of its good biocompatibility, biodegradability and non-toxic nature [56,57]. Pectin-based biodegradable hydrogels consist of pectin and polyacrylonitrile, have exhibited a $\mathrm{pH}$-responsive swelling-deswelling behavior at pH's 2 and 8 . This on- 
off switching behavior provides the hydrogel with the potential to serve as drug delivery vehicles [58]. Polymeric biomaterial based hydrogels viz., gelatin $(\mathrm{G})$ and sodium alginate $(\mathrm{SA})$ as base bio polymer(G/SA); or in combination with seabuckthron oil (SB) i.e., G/SA/SB; or polyvinylpyrrolidone (PVP) and carboxymethylcellulose (CMC) i.e., PVP/CMC; or with boric acid (BA) i.e., $\mathrm{PVP} / \mathrm{CMC} / \mathrm{BA}$ have the ability to be used as a tool for transdermal drug delivery [59].

20) Colloidosomes are made up of an aqueous or hydrogel core that is coated by a semi-permeable colloidal shell. Among several methodologies of colloidosome synthesis, it is generally performed by self-assembly of colloidal particles on the hydrogel core. The properties of the shell can be varied to control the rate of release of encapsulated drugs [60,61].

21) Thin film drug delivery vehicles are made up of hydrophilic polymers. They rapidly dissolve on the tongue or buccal cavity and delivers the drug(s) to the systemic circulation via dissolution when contact with liquid is made. Thin film drug delivery vehicles have emerged as an advanced alternative to the traditional tablets, capsules and liquids. Similar in size, shape and thickness to a postage stamp, thin film strips are typically designed for oral administration, with the user placing the strip on or under the tongue (sublingual) or along the inside of the cheek (buccal). These drug delivery options allow the medication to bypass the first pass metabolism thereby making the medication more bioavailable [62]. As the strip dissolves, the drug can enter the blood stream enterically, buccally or sublingually.

22) Fast dissolving tablet is designed for oral administration of solid dosage form of medicine in absence of fluid or water. Such tablets, when put on tongue dissolve in saliva instantaneously (within $60 \mathrm{sec}$ ) and release $\operatorname{drug}(\mathrm{s})$. They are found to be suitable during traveling where water may not be available $[63,64]$.

Recently, tablets with dual-delivery capabilities using "bi-layered dual-release technology" have been developed. Such tablets incorporate both an immediate-release and one or two controlled-release components having unique release profiles at different rates for one or more drugs [65].

23) Implantable drug delivery vehicles developed till date includes 1) Ceramic implants and 2) Silastic implants. Ceramic implants are made up of bioceramics. Among bioceramics, silica-based ordered mesoporous materials are unique candidates as bone implants due to two reasons: a) bioactive nature of such materials in contact with simulated body fluids, i.e., a carbonate hydroxyapatite similar to the mineral phase of bone is formed onto the material surfaces; b) their ability to act as delivery systems of many drugs to treat bone inflam- mation, infection, diseases and promote bone tissue regeneration using proteins, peptides, growth factors, and other osteogenic agents [66-69]. Therefore, in recent years, ceramic implants have gained attention as drug delivery carriers/ systems for bone regeneration therapies. On the other hand, silastic implants are made up of a silicon rubber membrane. These implants filled with drug(s) have been designed to provide a convenient and reliable method for the delivery of physiological concentrations of $\operatorname{drug}(\mathrm{s})$ viz., progesterone, nestorone [70,71]. These vehicles offer various advantages such as maintenance of therapeutic blood levels, improved patient compliance, and improved safety.

24) Controlled release formulations can be categorized into 3 subgroups: a) rate controlled release, b) delayed release, and c) pulse release formulations. Pulse release formulations are dealt with a branch of medicine called "chronotherapeutics". It deals with effects of biological clocks or circadian rhythms in human health [4]. It is a method of treatment where in vivo availability of drug is timed to match rhythms of disease. Therefore, this method optimizes therapeutic effects/ out comes of drug(s) and minimizes its side effects.

25) Drug loaded erythrocytes is one of the potential systems for targeted delivery of drugs, particularly to the reticulo-endothelial system. Erythrocytes are biocompatible, biodegradable, and possess long circulation halflife. They can be loaded with variety of biologically active substances. Through Erythrocyte Engineering, "carrier erythrocytes" are prepared by collecting blood sample from the organism of interest and separating RBCs from plasma. By using various physico-chemical methods, RBCs are systematically ruptured and the drug is entrapped into the erythrocytes, finally they are resealed and the resultant carriers are then called "resealed erythrocytes". Upon reinjection, the drug loaded erythrocytes serve as slow circulation depots, and prevents degradation of loaded drug(s) from inactivation by endogenous enzymes and chemicals. They provide steady state concentration of $\operatorname{drug}(\mathrm{s})$ and decrease the side-effects of loaded drug(s). Nowadays, through Erythrocyte Engineering, Nanoerythrosomes based drug delivery systems are being developed [72,73].

26) Self-emulsifying drug delivery systems (SEDDS) is an isotropic mixture of oils, surfactants and sometimes co-solvents. Self-emulsifying sustained/controlled-release pellets, capsules, suppositories and nanoparticles are being extensively investigated as drug delivery carriers. These multi-component delivery systems have been optimized by evaluating their ability to self-emulsify when introduced to an aqueous medium under gentle agitation, and by determination of particle size of the resulting emulsion. Upon oral administration, these systems form 
micro-emulsions in the gastrointestinal tract [74]. As drug delivery vehicles, SEDDS are best suited for the delivery of hydrophobic or lipophilic drugs. This selfemulsifying formulation greatly enhances the bioavailability and site-specific targeting of highly lipophilic drugs [75].

27) Research in micro-endoscopy is devoted to miniaturization of drug delivery devices/systems for intracorporeal use. In this approach, endoscopic function is increased by integrating its tip with Microsystems. The main aim of this research on diagnostic and therapeutic procedures is to reduce pain and discomfort and improve the quality of patient care. Recently, two groups of investigators have reported the development of endoscopic pills or endoscopic wireless devices [4].

28) Topical systems include phonophoresis and iontophoresis. Phonophoresis (a combination of ultrasound therapy and topical drug therapy) is a method of transporting drug(s) through skin using ultrasound (a sound wave outside the normal human hearing range). Now it is being used by physiotherapists to achieve therapeutic drug(s) concentrations at specific sites below skin [4]. Iontophoresis is an electro-chemical method of inducing migration of ionic drugs into skin by electrostatic repulsion at active electrode (by creating a potential gradient). Positively charged drugs are delivered by anode, whereas negatively charged ones by cathode. Its merits and limitations are well explained by Manabe and colleagues [76].

\section{Drug Delivery Routes}

The routes of administration of a drug(s) are mainly dependant on the properties of the drug(s) (for example, solubility, susceptibility to degradation by enzymes etc), access to a disease location, effectiveness in dealing with the specific disease and patient acceptability. The most preferred drug delivery route is the oral route. It is well known that most of the drugs which offer the greatest potential for more effective therapeutics are protein- and peptide-based. Therefore, they do not easily cross mucosal surfaces and biological membranes; they are easily denatured or degraded; prone to rapid metabolic clearance in the liver and other tissues of the body; and require precise dosing. At present, peptide, protein, antibody, vaccine and gene based drugs are generally administered by injection or a nanoneedle array [77]. This route is, however, less pleasant and also poses problems of oscillating blood-drug concentrations. Thus, despite the barriers to successful drug delivery that exists in the gastrointestinal tract, the oral route of drug administration is still the most preferred one; as it offers advantages of convenience and cheapness of administration. At present, drug(s) are also administered through other routes, such as topical (skin), transmucosal (buccal, sublingual, ocular, nasal, vaginal and rectal) and inhalation routes $[78,79]$.

1) Oral route of mucoadhesive drug delivery systems provides advantages of high accessibility and low enzymatic activity. Earlier the hydrophilic polymers (like SCMC, HPC and polycarbophil) were used for the treatment of periodontal diseases, but now attempts are being made to the effective utilization of these systems to the delivery of peptides, proteins and polysaccharides [80].

2) Parenteral routes include all routes which are other than oral. They are very promising and include subcutaneous, intravenous, intramuscular routes etc. Among nanomaterials, only liposomes have been administered intravenously. Nanoscale drug vehicles have been found to have great potential for improving the delivery of drugs through sublingual and nasal routes, as both of them avoid first-pass metabolism and for difficult-access brain, intra-articular and ocular cavities [77]. The active drug macromolecules are capable of associating with nanoparticles, thus making the systemic delivery of peptides and vaccines feasible through the nasal route. Moreover, there is a feasibility of improving the occular bioavailability of drugs if administered through a colloidal drug carrier [77]. Buccal and (or) sublingual routes of drug delivery uses a "dissolving film" or "oral drug strip" to administer drug(s) via absorption in the mouth and/or via the small intestines (enterically). Evaluating the systemic transmucosal drug delivery, the buccal mucosa is the preferred region as compared to the sublingual mucosa.

3) Trans-tissue and local delivery systems are needed to be firmly fixed to resected tissues during surgery with an aim to produce an enhanced pharmacological effect, while minimizing systemic, administration-associated toxicity. Trans-tissue systems include: drug-loaded gelatinous gels, which are formed in-situ and adhere to resected tissues, releasing drugs, proteins or gene-encoding adenoviruses; antibody-fixed gelatinous gels (cytokine barrier) that form a barrier, which, on a target tissue could prevent the permeation of cytokines into that tissue; cell-based delivery, which involves a gene-transduced oral mucosal epithelial cell (OMEC)-implanted sheet; device-directed delivery - a rechargeable drug infusion device that can be attached to the resected site.

4) Transdermal delivery of drug(s) is accompanied by formulations like transdermal patches, gels and sprays. A transdermal patch is placed on the skin to deliver a sustained release dose of medication through the skin. This system provides controlled release of the drug in the patient, and enables a steady blood-level profile, leading to reduced systemic side effects and, sometimes, improved efficacy over other dosage forms. A less elegant 
but effective alternative to a transdermal patch system is a conventional topical vehicle such as a gel. Such a formulation shows a clinically equivalent performance to that of a patch with lesser skin irritation and good compliance. Recently developed "Metered dose transdermal spray" (MDTS) is better alternative to both the patch and gel systems, as the sprays form the film that is quick drying, easy to use, well tolerated and non-occlusive. The MDTS formulations are topical aerosols formulated as single phase solutions consisting of drug, polymers and penetration enhancers [Axiron (testosterone transdermal metered-dose transdermal system) by Acrux/Eli Lilly] $[81,82]$. New metered dose transdermal sprays have broadened the patient acceptance and compliance. These methods can be used for symptoms of menopause, female androgen insufficiency, severe pain, central nervous system disorders, male androgen insufficiency, urinary incontinence, contraception and smoking cessation. Transdermal drug delivery systems have potential for systemic delivery of drugs with low oral bioavailability and high first pass effect. Transdermal delivery systems skip the problems of interference due to presence of food in the stomach, gastrointestinal modification (viz., hydrolytic, enzymatic, bacterial fermentation), metabolism, and variation in delivery rates. This technique is aesthetically accepted and usually non-invasive; and can be used to provide topical (local) delivery over weeks/ months. It is suitable for unconscious patients but suffers from lack of dose flexibility, slow penetration rates and restricted to low dosage drugs [4].

5) Pulmonary delivery of $\operatorname{drug}(\mathrm{s})$ is accomplished by aerosols, nebulizers (solutions), powders (dry powder inhaler, DPIs), and metered-dose inhalers (MDIs). All of these utilize nanostructures like dendrimers, micelles, nanoparticles etc. Pulmonary drug delivery systems have been successfully used for local targeting for the treatment of respiratory diseases, as well it has the potential for effective delivery of numerous drugs systemically, as explicitely explained by Shaikh and coworkers [83].

6) For gene delivery, the plasmid DNA must be introduced into the host cells, followed by its transcription and translation into the corresponding protein. To achieve this goal, few hurdles must be overcome by the gene delivery system as the transfection is affected by: 1) targeting the delivery system to the host cell, 2) transport through the cell membrane, 3) uptake and degradation in the endolysosomes, and 4) intracellular trafficking of plasmid DNA to the nucleus. Therefore, the delivery of genes for treating genetic diseases still remains a challenge.

7) Acoustic targeted drug delivery (ATDD) is a method that uses ultrasound energy to enhance the transport of molecules into and (or) across specific tis- sues [84]. Generally, this class of ultrasound energy falls under the class of therapeutic ultrasound, and ranges in ultrasonic frequencies of $1-20 \mathrm{MHz}$. The use of ATDD in conjunction with local drug delivery by injection, topical application and convection-enhanced delivery shows promise to significantly enhance the treatment of various diseases in the human body by specifically targeting the drug(s) into the tissue [85].

\section{Applications of Drug Delivery Technology}

Significant changes in the healthcare industry have led pharmaceutical companies to depend mainly on advanced drug delivery technology to produce distinctive drugs and remain competitive. In addition, soaring R\&D costs, long timeframes for developing drugs from new chemical entities, and increasing consumer demand for better, more convenient medicines, pharmaceutical companies are using creative strategies that capitalize on the capabilities of drug delivery technology. Therefore, a growing number of pharmaceutical companies are now applying the technologies of drug-delivery companies to enhance the convenience, safety, and efficacy of currently marketed drugs and drug products. Drug delivery companies, in turn, are developing more sophisticated technologies to meet new demands of patients and the pharmaceutical industry. Against this background, innovative pharmaceutical companies are partnering with drug delivery companies to find new ways of developing advanced medications to meet these market demands. Therefore, they are employing lower-risk strategies to develop existing medicines into improved therapies rapidly, with lower development costs and attrition rates. For example, a UK-based pharmaceutical company named "Arakis Limited" formed a strategic partnership with US-based Penwest (an oral drug delivery and development company) to develop 'Performance-Enhanced Medicines' (PEMs). PEMs are drugs that have been substantially improved, clinically and commercially, by drug delivery technology. The strategy for producing PEMs is creatively simple. Arakis takes advantage of new discoveries in medical science and genomics to identify and acquire existing drugs that can be improved through drug delivery to satisfy the unmet medical needs of specific disease populations. Patient genotyping and metabolic profiling are valuable tools for selecting specific patient populations in which a drug will be particularly effective. Thus, successful development of PEMs improve the drug's therapeutic benefit, efficacy, safety, delivery profile or dosage, and reduce side effects.

The applications of fine particle approach based drug delivery technology to the pharmaceutical industries have the potential to:

1) extend patent life of existing drugs through re-for- 
mulation based upon fine-particle platform;

2) improve the likelihood of development success for new compounds and novel agents which face a) water solubility problems, b) protein and peptide drugs delivery problems by stabilizing them through Supercritical Fluid (SCF) Technology [86-90];

3) improve effectiveness of a drug and/or device through coating;

4) help solve drug delivery problems.

Thus, application of drug delivery technology to pharma companies provides immense opportunity to enhance value for currently marketed drugs, drug-related products.

\section{Market Scenario of Drug Delivery Systems}

Recent report of the major types of drug delivery systems used in the healthcare industry and comprehensive coverage of the pharmaceuticals markets has documented that the global market for advanced drug delivery systems amounted to $\$ 139$ billion in 2009. The estimate for 2014 is $\$ 196.4$ billion, for a compound annual growth rate (CAGR) of $7.2 \%$ in the 5 -year period. The largest segment of the market is targeted drug delivery $(\$ 50.9$ billion in 2009) which is expected to increase to $\$ 80.2$ billion in 2014, for a CAGR of $9.5 \%$. Sustained-release products have the second largest market share (\$ 36.1 billion in 2009) and are expected to rise to $\$ 45.8$ billion in 2014, for a CAGR of $4.9 \%$ [91].

\section{Conclusions with Future Opportunities and Challenges}

In nutshell, majority of recent advances in drug delivery systems are primarily based on the nanotechnology. Nanomaterials and nanoformulations provide immense benefits to the drug delivery, targeting, release and have additional potential to combine diagnosis and therapy. Therefore, they are emerging successfully as one of the major tools in nanomedicine.

Nanomaterials based drug delivery systems have greater potential for many applications, like AIDS therapy, anti-tumor therapy, gene therapy, radiotherapy, in the delivery of antibiotics, proteins, vaccines, virostatics, and as vesicles to pass the blood-brain barrier. The main goals are to improve their stability in the biological environment, to mediate the bio-distribution of active compounds, improve drug loading, targeting, transport, release, and interaction with biological barriers. In certain cases, cytotoxicity of nanomaterials or their degradation products remains a major problem. Thus, improvements in biocompatibility of such materials are major concern of future research. Moreover, there are several technological challenges to be overcome, in developing nano- materials based drug delivery systems:

1) Those delivering highly localized, large quantities of $\operatorname{drug}(\mathrm{s})$ to specific sites must release $\operatorname{drug}(\mathrm{s})$ in controlled ways;

2) Controllable release profiles must be obtained for sensitive drug(s);

3) Nanomaterials must be made up of biocompatible and biodegradable substances;

4) Structures should be like biomimetic polymers and nanotubes;

5) Should function as active drug targeting/on-command delivery/systems interacting with the body/targeted or smart drug delivery/ intelligent drug release devices or bioresponsive triggered systems/self-regulated delivery systems;

6) Must be virus-like systems for intracellular delivery;

7) Must have nanoparticles to improve devices such as implantable devices/nanochips for nanoparticle release, or multi-reservoir drug delivery-chips;

8) Nanoparticles must be made for tissue engineering; e.g. for the delivery of cytokines to control cellular growth and differentiation, and stimulate regeneration; or for coating implants with nanoparticles in biodegradable polymer layers for sustained release;

9) Advanced polymeric carriers for the delivery of therapeutic peptide or proteins or biopharmaceutics;

10) In the development of Combined therapy and medical imaging, for e.g., nanoparticles for diagnosis and manipulation during surgery (e.g. thermotherapy with magnetic particles);

11) Universal formulation schemes that can be used as intravenous, intramuscular or oral drugs;

12) Cell and gene targeting systems;

13) Must be user-friendly lab-on-a-chip devices for point-of-care and disease prevention and control at home;

14) Devices for detecting changes in magnetic or physical properties after specific binding of ligands on paramagnetic nanoparticles that can correlate with the amount of ligand;

15) Must serve as better disease markers in terms of sensitivity and specificity;

16) Insolubility issues of the past have provoked a paradigm change, which now offered a novel solution called SEDDS which needs further exploration and research so as to bring out commercially available selfemulsifying formulations.

Apart from these, developing oral drug delivery vehicles for e.g. "oral strips" need plasticizers in its formulations. However, plasticizers are not free from health hazards, as $90 \%$ of the plasticizers belong to a family of industrial compounds called "Phthalates". All of the phthalates are ubiquitous environmental contaminants or per- 
sistent organic pollutants (POP) or POP-like compounds, as well linked to a range of adverse reproductive effects [92-95]. Therefore, utmost care must be taken about any adverse health impact associated with the organic or inorganic elements and compounds used in developing any drug delivery systems.

\section{Acknowledgements}

We are very much thankful to the anonymous reviewer(s) for providing valuable comments on this review article.

\section{REFERENCES}

[1] V. P. Torchilin, "Lipid-Core Micelles for Targeted Drug Delivery," Current Drug Delivery, Vol. 2, No. 4, 2005, pp. 319-327. doi:10.2174/156720105774370221

[2] V. P. Torchilin, "Micellar Nanocarriers: Pharmaceutical Perspectives," Pharmaceutical Research, Vol. 24, No. 1, 2007, pp. 1-16. doi:10.1007/s11095-006-9132-0

[3] R. R. Sawant and V. P. Torchilin, "Polymeric Micelles: Polyethylene Glycol-Phosphatidylethanolamine (PEG-PE)Based Micelles as an Example," Methods in Molecular Biology, Vol. 624, 2010, pp. 131-149. doi:10.1007/978-1-60761-609-2_9

[4] P. D. Reddy and D. Swarnalatha, "Recent Advances in Novel Drug Delivery Systems," International Journal of PharmTech Research, Vol. 2, No. 3, 2010, pp. 20252027.

[5] M. Jahanshahi and Z. Babaei, "Protein Nanoparticle: A Unique System as Drug Delivery Vehicles," African Journal of Biotechnology, Vol. 7, No. 25, 2008, pp. 49264934.

[6] F. Scherer, M. Anton, U. Schillinger, J. Henke, C. Bergemann, A. Krager, B. Gonsbacher and C. Plank, "Magnetofection: Enhancing and Targeting Gene Delivery by Magnetic Force in Vitro and in Vivo," Gene Therapy, Vol. 9, No. 2, 2002, pp. 102-109. doi:10.1038/sj.gt.3301624

[7] J. Dobson, "Gene Therapy Progress and Prospects: Magnetic Nanoparticle-Based Gene Delivery," Gene Therapy, Vol. 13, 2006, pp. 283-287. doi:10.1038/sj.gt.3302720

[8] M. Mahmoudi, A. Simchi and M. Imani, "Recent Advances in Surface Engineering of Superparamagnetic Iron Oxide Nanoparticles for Biomedical Applications," Journal of Iranian Chemical Society, Vol. 7, 2010, pp. S1S27.

[9] K.-O. Kim, B.-S. Kim and I.-S. Kim, "Self-Ssembled Core-Shell Poly(EthyleneGlycol)-POSS Nanocarriers for Drug Delivery," Journal of Biomaterials and Nanobiotechnology, Vol. 2, No. 3, 2011, pp. 201-206. doi:10.4236/jbnb.2011.23025

[10] L. W. Thomas, D. T. Simpson, T. N. Val, Z. Yu, K. T. Lim, E. J. Park, R. O. Williams III and K. P. Johnston, "Encapsulation of Protein Nanoparticles into UniformSized Microspheres Formed in a Spinning Oil Film," AAPS PharmSciTech, Vol. 6, No. 4, 2005, pp. E605E617.
[11] A. Chonn and P. R. Cullis, "Recent Advances in Liposomal Drug-Delivery Systems," Current Opinion in Biotechnology, Vol. 6, No. 6, 1995, pp. 698-708. doi:10.1016/0958-1669(95)80115-4

[12] J. Kunisawa, T. Masuda, K. Katayama, T. Yoshikawa, Y. Tsutsumi, M. Akashi, T. Mayumi and S. Nakagawa, "Fusogenic Liposome Delivers Encapsulated Nanoparticles for Cytosolic Controlled Gene Release," Journal of Controlled Release, Vol. 105, No. 3, 2005, pp. 344-353. doi:10.1016/j.jconrel.2005.03.020

[13] D. J. Bharali, M. Khalil, M Gurbuz, T. M. Simone and S. A. Mousa, "Nanoparticles and Cancer Therapy: A Concise Review with Emphasis on Dendrimers," International Journal of Nanomedicine, Vol. 4, 2009, pp. 1-7.

[14] J. E. Adrian, H. W. Morselt, R. Süss, S. Barnert, J. W. Kok, S. A. Asgeirsdóttir, M. H. Ruiters, G. Molema and J. A. Kamps, "Targeted SAINT-O-Somes for Improved Intracellular Delivery of siRNA and Cytotoxic Drugs into Endothelial Cells," Journal of Controlled Release, Vol. 144, No. 3, 2010, pp. 341-349. doi:10.1016/j.jconrel.2010.03.003

[15] A. Csiszar, N. Hersch, S. Dieluweit, R. Biehl, R. Merkel and B. Hoffmann, "Novel Fusogenic Liposomes for Fluorescent Cell Labeling and Membrane Modification," Bioconjugate Chemistry, Vol. 21, No. 3, 2010, pp. 537543. doi:10.1021/bc900470y

[16] M. Ferrari, "Cancer Nanotechnologies: Opportunities and Challenges," Nature Reviews: Cancer, Vol. 5, No. 3, 2005, pp. 161-171. doi:10.1038/nrc1566

[17] H. S. Sharma, D. F. Muresanu, A. Sharma, R. Patnaik and J. V. Lafuente, "Chapter 9-Nanoparticles Influence Pathophysiology of Spinal Cord Injury and Repair," Progress in Brain Research, Vol. 180, 2009, pp. 154-180. doi:10.1016/S0079-6123(08)80009-X

[18] H. S. Sharma, S. F. Ali, Z. R. Tian, R. Patnaik, S. Patnaik, A. Sharma, A. Boman, P. Lek, E. Seifert and T. Lundstedt, "Nanowired-Drug Delivery Enhances Neuroprotective Efficacy of Compounds and Reduces Spinal Cord Edema Formation and Improves Functional Outcome Following Spinal Cord Injury in the Rat," Acta Neurochir Supplement, Vol. 106, 2010, pp. 343-350. doi:10.1007/978-3-211-98811-4_63

[19] K. E. Fischer, B. J. Alemán, S. L. Tao, R. Hugh Daniels, E. M. Li, M. D. Bünger, G. Nagaraj, P. Singh, A. Zettl and T. A. Desai, "Biomimetic Nanowire Coatings for Next Generation Adhesive Drug Delivery Systems," Nanotechnology Letters, Vol. 9, No. 2, 2009, pp. 716720.

[20] H. Liou, H. Lin, Y. Hwu, W. Chen, W. Liou, L. Lai, W. Lin and W. Chiou, "Synthesis and Characterization of Novel Hybrid Poly(methyl methacrylate)/Iron Nanowires for Potential Hyperthemia Therapy," Journal of Biomaterials and Nanobiotechnology, Vol. 1, 2010, pp. 50-60. doi:10.4236/jbnb.2010.11007

[21] G. A. Hughes, "Nanostructure-Mediated Drug Delivery," Nanomedicine: Nanotechnology, Biology and Medicine, Vol. 1, No. 1, 2005, pp. 22-30. 
doi:10.1016/i.nano.2004.11.009

[22] M. Bikram, A. M. Gobin, R. E. Whitmire and J. L. West, "Temperature-Sensitive Hydrogels with $\mathrm{SiO}_{2}-\mathrm{Au}$ Nanoshells for Controlled Drug Delivery," Journal of Controlled Release, Vol. 123, No. 3, 2007, pp. 219-227. doi:10.1016/j.jconrel.2007.08.013

[23] I. Bob, "Nanoparticle Drug Delivery Systems," Innovations in Pharmaceutical Technology, Vol. 24, 2007, pp. 58-62.

[24] M. M. De Villiers and Y. M. Lvov, "Nanoshells for Drug Delivery," Nanotechnologies for the Life Sciences, Published Online 15 September 2007.

[25] K. Noh, K. S. Brammer, C. Choi, S. Kim, C. J. Frandsen and S. Jin, "A New Nano-Platform for Drug Release via Nanotubular Aluminum Oxide," Journal of Biomaterials and Nanobiotechnology, Vol. 2, 2011, pp. 226-233. doi:10.4236/jbnb.2011.23028

[26] R. J. Chen and D. Hongjie, "Noncovalent Functionalization of Carbon Nanotubes for Highly Specific Electronic Biosensors," Proceedings of National Academy of Sciences, USA, Vol. 100, No. 9, 2003, pp. 4984-4989.

[27] A. Woolley, et al., "Direct Haplotyping of Kilobase-Size DNA Using Carbon Nanotube Probes," Nature Biotechnology, Vol. 18, 2000, pp. 760-764. doi:10.1038/77760

[28] A. L. Yarin, "Nanofibers, Nanofluidics, Nanoparticles and Nanobots for Drug and Protein Delivery Systems," Scientia Pharmaceutica, Vol. 78, 2010, p. 542. doi:10.3797/scipharm.cespt.8.L02

[29] "Nanoneedles Make Injections Painless," http://www.in-pharmatechnologist.com/Materials-Formul ation/Nano-needles-make-injections-painless

[30] T. Vieru, "Nanoneedles May Change the Face of Medicine," Accessed on 29 April 2009.

http://news.softpedia.com/news/Nanoneedles-May-Chang e-the-Face-of-Medicine-110439.shtml

[31] A. Lewcock, "Carbon Nanoneedles for Drug Delivery," Accessed on 8 February 2007. http://www.in-pharmatechnologist.com/Materials-Formul ation/Carbon-nanoneedles-for-drug-delivery

[32] H. Chen, J. Han, J. Li and M. Meyyappan, "Microelectronic DNA Assay for the Detection of BRCA1 Gene Mutations," Biomedical Microdevices, Vol. 6, No. 1, 2004, pp. 55-60.

doi:10.1023/B:BMMD.0000013366.85609.dd

[33] S. Sengupta and R. Sasisekharan, "Exploiting Nanotechnology to Target Cancer," British Journal of Cancer, Vol. 96, No. 9, 2007, pp. 1315-1319. doi:10.1038/sj.bjc.6603707

[34] P. A. Shashishekar, C. Jin, L. A. Curtiss, Nancy A. Monteiro-Riviere and R. J. Narayan, "Nanoporous Membranes for Medical and Biological Applications," WIREs Nanomedicine and Nanobiotechnology, Vol. 1, No. 5, 2009, pp. 568-581. doi:10.1002/wnan.50

[35] T. Lebold, C. Jung, J. Michaelis and C. Brauchle, "Nanostructured Silica Materials as Drug-Delivery Systems for Doxorubicin: Single Molecule and Cellular Studies," Nano Letters, Vol. 9, No. 8, 2009, pp. 2877-
2883. doi: $10.1021 / \mathrm{n} 19011112$

[36] N. Darshana, K. S. Rathore, M. Bharkatiya, S. S. Sisodia and R. K. Nema, "Bucky Balls: A Novel Drug Delivery System," Journal of Chemical and Pharmaceutical Research, Vol. 2, No. 2, 2010, pp. 240-248.

[37] W. C. W. Chan, "Bio-Applications of Nanoparticles," Landes Bioscience, Austin, USA, 2007.

[38] C. A. Mirkin, R. L. Letsinger, R. C. Mucic and J. J. Storhoff, "A DNA Based Method for Rationally Assembling Nanoparticles into Macroscopic Materials," Nature, Vol. 382, No. 6592, 1996, pp. 607-609. doi:10.1038/382607a0

[39] G. F. Paciotti, M. Lonnie, D. Weinreich, D. Goia, N. Pavel, R. E. McLaughlin and T. Lawrence, "Colloidal Gold: A Novel Nanoparticle Vector for Tumor Directed Drug Delivery," Drug Delivery, Vol. 11, No. 3, 2004, pp. 169-183. doi:10.1080/10717540490433895

[40] X. Wu and M. P. Bruchez, "Immunofluorescent Labeling of Cancer Marker Her2 and Other Cellular Targets with Semiconductor Quantum Dots," Nature Biotechnology, Vol. 21, 2003, pp. 41-46. doi:10.1038/nbt764

[41] J. K. Jaiswal and S. M. Simon, "Long-Term Multiple Color Imaging of Live Cells Using Quantum Dot Bioconjugates," Nature Biotechnology, Vol. 21, No. 1, 2003, pp. 47-51. doi:10.1038/nbt767

[42] X. H. Gao and S. M. Nie, "Molecular Profiling of Single Cells and Tissue Specimens with Quantum Dots," Trends in Biotechnology, Vol. 21, No. 9, 2003, pp. 371-373. doi:10.1016/S0167-7799(03)00209-9

[43] T. M. Jovin, "Quantum Dots Finally Come of Age," Nature Biotechnology, Vol. 21, 2003, pp. 32-33. doi:10.1038/nbt0103-32

[44] S. K. Vashist, R. Tewari, R. P. Bajpai, L. M. Bharadwaj and R. Roberto, "Review of Quantum Dot Technologies for Cancer Detection and Treatment," Journal of Nanotechnology, Online, 13 September 2006.

[45] L. Qi and X. Gao, "Emerging Application of Quantum Dots for Drug Delivery and Therapy," Expert Opinion in Drug Delivery, Vol. 5, No. 3, 2008, pp. 263-267. doi:10.1517/17425247.5.3.263

[46] A. Bianco, K. Kostarelos and M. Prato, "Applications of Carbon Nanotubes in Drug Delivery," Current Opinion in Chemical Biology, Vol. 9, No. 6, 2005, pp. 674-679. doi:10.1016/j.cbpa.2005.10.005

[47] L. Lacerda, A. Bianco, M. Prato and K. Kostarelos, "Carbon Nanotubes as Nanomedicines: From Toxicology to Pharmacology," Advanced Drug Delivery Reviews, Vol. 58, No. 14, 2006, pp. 1460-1470. doi:10.1016/j.addr.2006.09.015

[48] C. Klumpp, K. Kostarelos, M. Prato and A. Bianco, "Functionalized Carbon Nanotubes as Emerging Nanovectors for the Delivery of Therapeutics," Biochimica et Biophysica Acta-Biomembranes, Vol. 1758, No. 3, 2006, pp. 404-412.

[49] N. W. Kam, M. O’Connell, J. A. Wisdom and H. Dai, "Carbon Nanotubes as Multifunctional Biological Transporters and Near-Infrared Agents for Selective Cancer 
Cell Destruction," Proceedings of National Academy of Sciences, USA, Vol. 102, No. 33, 2005, pp. 11600-11605. doi:10.1073/pnas.0502680102

[50] M. Lou and E. Jonckheere, "Magnetically Levitated Nano- robots: An Application to Visualization of Nerve Cells Injuries," Studies on Health Technology and Information, Vol. 125, 2007, pp. 310-312.

[51] Nanotech Web, Accessed on 15 January 2009. http://nanotechweb.org/cws/article/lab/32077

[52] A. Rastogi, T. Bose, M. D. Feldman, D. Patel and S. Stavchansky, "Characterization of Nanoporous Surfaces as Templates for Drug Delivery Devices," Journal of American Association of Pharmaceutical Scientists, Vol. 11, 2009, pp. 758-761.

[53] Y. Cheng, J. Wang, T. Rao, X. He and T. Xu, "Pharmaceutical Applications of Dendrimers: Promising Nanocarriers for Drug Delivery," Frontiers in Biosciences, Vol. 13, 2008, pp. 1447-1471. doi:10.2741/2774

[54] A. Samad, M. I. Alam and K. Saxena, "Dendrimers: A Class of Polymers in the Nanotechnology for the Delivery of Active Pharmaceuticals," Current Pharmaceuticals Design, Vol. 15, No. 25, 2009, pp. 2958-2969. doi:10.2174/138161209789058200

[55] J. Kopecek, "Smart and Genetically Engineered Biomaterials and Drug Delivery Systems," European Journal of Pharmaceutical Sciences, Vol. 20, No. 1, 2003, pp. 1-16. doi:10.1016/S0928-0987(03)00164-7

[56] S. A. Agnihotri, N. N. Mallikarjuna and T. M. Aminabhavi, "Recent Advances on Chitosan-Based Micro- and Nanoparticles in Drug Delivery," Journal of Controlled Release, Vol. 100, No. 1, 2004, pp. 5-28. doi:10.1016/i.jconrel.2004.08.010

[57] P. Tangri and N. V. Satheesh Madhav, "Recent Advances in Oral Mucoadhesive Drug Delivery Systems: A Review," (Online www.ijprd.com), Publication Ref No.: IJPRD/ 2011/PUB/ARTI/VOV-3/ISSUE-2/APRIL/018; ISSN 0974-9446.

[58] M. Sadeghi, "Pectin-Based Biodegradable Hydrogels with Potential Biomedical Applications as Drug Delivery Systems," Journal of Biomaterials and Nanobiotechnology, Vol. 2, 2011, pp. 36-40. doi:10.4236/jbnb.2011.21005

[59] N. Saha, A. Saarai, N. Roy, T. Kitano and P. Saha, "Polymeric Biomaterial Based Hydrogels for Biomedical Applications," Journal of Biomaterials and Nanobiotechnology, Vol. 2, 2011, pp. 85-90. doi:10.4236/jbnb.2011.21011

[60] A. D. Dinsmore, M. F. Hsu, M. G. Nikolaides, M. Marquez, A. R. Bausch and D. A. Weitz, "Colloidosomes: Selectively Permeable Capsules Composed of Colloidal Particles," Science, Vol. 298, No. 5595, 2002, pp. 10061009. doi:10.1126/science. 1074868

[61] R. T. Rosenberg and N. Dan, "Self-Assembly of Colloidosome Shells on Drug-Containing Hydrogels," Journal of Biomaterials and Nanobiotechnology, Vol. 2, 2011, pp. 1-7. doi:10.4236/jbnb.2011.21001
[62] “Oral Thin Films," In: Orally Disintegrating Tablet and Film Technologies, 5th Edition, Technology Catalysts International, Falls Church, VA, 2008.

[63] S. K. Singh, D. N. Mishra, R. Jassal and P. Soni, "Fast Disintegrating Combination Tablets of Omeprazole, and Domperidone," Asian Journal of Pharmaceutical and Clinical Research, Vol. 2, No. 3, 2009, pp. 74-82.

[64] D. Bhowmik, B. Chiranjib, Krishnakanth, Pankaj and R. M. Chandira, "Fast Dissolving Tablet: An Overview," Journal of Chemical and Pharmaceutical Research, Vol. 1, No. 1, 2009, pp. 163-177.

[65] A. Baichwall, "Culturing Innovation and Enhancing Medications Using Oral Drug Delivery," Drug Development and Delivery, Vol. 2, 2002. http://www.drugdeliverytech.com/ME2

[66] I. I. Slowing, B. G. Trewyn and V. S.-Y. Lin, "Mesoporous Silica Nanoparticles for Intracellular Delivery of Membrane-Impermeable Proteins," Journal of American Chemical Society, Vol. 129, No. 28, 2007, pp. 8845-8849. doi:10.1021/ja0719780

[67] F. Balas, M. Manzano, M. Colilla, et al., " $L$-Trp Absorption into Silica Mesoporous Materials to Promote Bone Formation," Acta Biomaterialia, Vol. 4, No. 3, 2008, pp. 514-522. doi:10.1016/j.actbio.2007.11.009

[68] M. Colilla, M. Manzano and M. Vallet-Regi, "Recent Advances in Ceramic Implants as Drug Delivery Systems for Biomedical Applications," International Journal of Nanomedicine, Vol. 3, No. 4, 2008, pp. 403-414.

[69] M. Vallet-Regi, M. Colilla and I. Izquierdo-Barba, "Bioactive Mesoporous Silica as Controlled Delivery Systems: Application in Bone Tissue Regeneration," Journal of Biomedical Nanotechnology, Vol. 4, No. 3, 2008, pp. $1-15$.

[70] S. R. Milligan and P. E. Cohen, "Silastic Implants for Delivering Physiological Concentrations of Progesterone to Mice," Reproduction Fertility and Development, Vol. 6, No. 2, 1994, pp. 235-239. doi:10.1071/RD9940235

[71] R. Sitruk-Ware, M. Small, N. Kumar, Y. Y. Tsong, K. Sundaram and T. Jackanicz, "Nestorone: Clinical Applications for Contraception and HRT," Steroids, Vol. 68, No. 10, 2003, pp. 907-913. doi:10.1016/S0039-128X(03)00140-5

[72] W. E. Lynch, G. P. Sartiano and A. Ghaffar, "Erythrocytes as Carriers of Chemotherapeutic Agents for Targeting the Reticuloendothelial System," American Journal of Hematology, Vol. 9, No. 3, 1980, pp. 249-259. doi:10.1002/ajh.2830090303

[73] P. D. Patel, N. Dand, R. S. Hirlekar and V. J. Kadam, "Drug Loaded Erythrocytes: As Novel Drug Delivery System," Current Pharmaceutical Design, Vol. 14, No. 1, 2008, pp. 63-70. doi:10.2174/138161208783330772

[74] A. Pathak, V. Jain, A. K. Nagariya, R. Singh, S. Nayak, P. Bansal, V. Gupta, S. Kumar and H. Singh, "Recent Advances in Self Emulsifying Drug Delivery System-A Review," Drug Invention Today, Vol. 2, No. 2, 2010, pp. 123-129. 
[75] A. Spernath and A. Aserin, "Microemulsions as Carriers for Drugs and Nutraceuticals," Advances in Colloid Interface Sciences, Vol. 128-130, 2006, pp. 47-64. doi:10.1016/j.cis.2006.11.016

[76] T. Manabe, H. Okino, R. Maeyama, K. Mizumoto, E. Nagai, et al., "Novel Strategic Therapeutic Approaches for Prevention of Local Recurrence of Pancreatic Cancer after Resection: Trans-Tissue, Sustained Local DrugDelivery Systems," Journal of Controlled Release, Vol. 100, No. 3, 2004, pp. 317-330. doi:10.1016/i.jconrel.2004.09.007

[77] C. B. Packhaeuser, J. Schnieders, C. G. Oster and T. Kissel, "In Situ Forming Parenteral Drug Delivery Systems: An Overview," European Journal of Pharmaceutics and Biopharmaceutics, Vol. 58, No. 2, 2004, pp. 445-455. doi:10.1016/i.ejpb.2004.03.003

[78] M. Cobleigh, V. K. Langmuir, G. W. Sledge, K. D. Miller, L. Haney, et al., "A Phase I/II Dose-Escalation Trial of Bevacizumab in Previously Treated Metastatic Breast Cancer," Seminars in Oncology, Vol. 30, No. 5, 2003, pp. 117-124. doi:10.1053/j.seminoncol.2003.08.013

[79] V. P. Torchilin, "Multifunctional Nanocarriers," Advances in Drug Delivery Reviews, Vol. 58, No. 14, 2006, pp. 1532-1555. doi:10.1016/j.addr.2006.09.009

[80] G. P. Andrews, et al., "Mucoadhesive Polymeric Platforms for Controlled Drug Delivery," European Journal of Pharmaceutics and Biopharmaceutics, Vol. 71, No. 3, 2009, pp. 505-518. doi:10.1016/j.eipb.2008.09.028

[81] I. S. Fraser, E. Weisberg, N. Kumar, A. J. Humberstone, L. McCrossin, et al., "An Initial Pharmacokinetic Study with a Metered Dose Transdermal System for Delivery of the Progestogen Nestorone as a Possible Future Contraceptive," Contraception, Vol. 76, No. 6, 2007, pp. 432438. doi:10.1016/j.contraception.2007.08.006

[82] A. Bakshi, A. Bajaj, G. Malhotra, M. Madan and N. Amrutiya, "A Novel Metered Dose Transdermal Spray Formulation for Oxybutynin," Indian Journal of Pharmaceutical Sciences, Vol. 70, No. 6, 2008, pp. 733-739. doi:10.4103/0250-474X.49094

[83] S. Shaikh, S. Nazim, T. Khan, A. Shaikh, A. Zameeruddin and A. Quazi, "Recent Advances in Pulmonary Drug Delivery System: A Review," International Journal of Applied Pharmaceutics, Vol. 2, 2010, pp. 27-31.

[84] Lewis Jr., et al., "Acoustic Targeted Drug Delivery in Neurological Tissue," 2007.

[85] Lewis Jr., et al., "Acoustic Chemotherapy and Brain Cancer," 2007. http://www.acoustics.org/press/154th/lewis.html

[86] N. Jovanović, A. Bouchard, G. W. Hofland, G. J. Witkamp, D. J. Crommelin and W. Jiskoot, "Stabilization of
Proteins in Dry Powder Formulations Using Supercritical Fluid Technology," Pharmaceutical Research, Vol. 21, No. 11, 2004, pp. 1955-1969. doi:10.1023/B:PHAM.0000048185.09483.e7

[87] A. R. Duarte, M. S. Costa, A. L. Simplício, M. M. Cardoso and C. M. Duarte, "Preparation of Controlled Release Microspheres Using Supercritical Fluid Technology for Delivery of Anti-Inflammatory Drugs," International Journal of Pharmacology, Vol. 308, 2006, pp. 168-174. doi:10.1016/j.ijpharm.2005.11.012

[88] K. Byrappa, S. Ohara and T. Adschiri, "Nanoparticles Synthesis Using Supercritical Fluid Technology-Towards Biomedical Applications," Advanced Drug Delivery Reviews, Vol. 60, No. 3, 2008, pp. 299-327. doi:10.1016/j.addr.2007.09.001

[89] T. Yasuji, H. Takeuchi and Y. Kawashima, "Particle Design of Poorly Water-Soluble Drug Substances Using Supercritical Fluid Technologies," Advanced Drug Delivery Reviews, Vol. 60, No. 3, 2008, pp. 388-398. doi:10.1016/j.addr.2007.03.025

[90] S. P. Cape, J. A. Villa, E. T. S. Huang, T. Yang, J. F. Carpenter and R. E. Sievers, "Preparation of Active Proteins, Vaccines and Pharmaceuticals as Fine Powders using Supercritical or Near-Critical Fluids," Pharmaceutical Research, Vol. 25, No. 9, 2008, pp. 1967-1990. doi:10.1007/s11095-008-9575-6

[91] BCC Research LLC, 49 Walnut Park, Building 2, Wellesley, MA 02481, USA. http://www.bccresearch.com/report/PHM006G.html

[92] B. C. Blount, M. J. Silva, S. P. Caudill, L. L. Needham, J. L. Prikle, E. J. Sampson, G. W. Lucier, R. J. Jackson and J. W. Brock, "Levels of Seven Urinary Phthalate Metabolites in a Human Reference Population," Environmental Health Perspectives, Vol. 108, No. 10, 2000, pp. 979-982. doi:10.1289/ehp.00108979

[93] NTP-CERHR, "Monograph on the Potential Human Reproductive and Developmental Effects of Di-n-Butyl Phthalate (DBP)," NTP CERHR MON, 2003, Apr(4), i-III90.

[94] H. M. Koch, H. Drexler and J. Angerer, "Internal Exposure of Nursery-School Children and Their Parents and Teachers to Di-(2-ethyl hexyl) Phthalate (DEHP)," International Journal of Hygiene and Environmental Health, Vol. 207, No. 1, 2004, pp. 15-22. doi:10.1078/1438-4639-00270

[95] S. H. Swan, K. M. Main, F. Liu, S. L. Stewart, R. L. Kruse, et al., "Decrease in Anogenital Distance among Male Infants with Prenatal Phthalate Exposure," Environmental Health Perspectives, Vol. 113, 2005, pp. 10561061. doi:10.1289/ehp. 8100 